\title{
Wirksamkeit von Lernarrangements in der kaufmännischen Erstausbildung - Zur Bedeutung von Vorwissen und Bildungsabschlüssen
}

\author{
Jürgen Seifried
}

\section{Zusammenfassung}

Untersucht wird, wie sich unterschiedliche Bildungsabschlüsse auf die Kompetenzentwicklung in der kaufmännischen Erstausbildung niederschlagen. Vor dem Hintergrund methodischer Grundentscheidungen (schüler- vs. lehrerzentrierte Arrangements) wird gezeigt, wie Schüler mit unterschiedlichen Bildungsabschlüssen die kaufmännische Erstausbildung erleben und welche Lernerfolge sie erzielen. Die Datenbasis der Auswertungen bilden drei längsschnittlich angelegte quasi-experimentelle Feldstudien, bei denen Experimentalgruppen (Selbstorganisiertes Lernen) jeweils nach dem Muster des fragend-entwickelnden Unterrichts einer Kontrollgruppe gegenübergestellt wurden (Prozessanalysen Selbstorganisierten Lernens).

\section{Zur Diskussion um „Ausbildungsfähigkeit“}

Die derzeitige Diskussion über die Probleme des Bildungswesens lässt Zweifel aufkommen, ob die Funktionen der Qualifikation, Integration und Legitimation sowie Selektion und Allokation (vgl. Fend 1981) ausreichend gut erfüllt werden. Bildungs- und Qualifikationsdefizite werden spätestens dann spürbar, wenn Jugendliche und junge Erwachsene in die Berufsausbildung einmünden. Vielstimmig wird an dieser ersten Schwelle über fehlende "Ausbildungsfähigkeit“ geklagt. Empirische Studien bei Schulabgängern zeigen, dass diese Klagen durchaus gerechtfertig sind. Eine Untersuchung zu den Kompetenzprofilen Berliner Jugendlicher beim Eintritt in das Berufsbildungssystem belegt gravierende Defizite (vgl. Badel 2003). Ein beträchtlicher Teil der Jugendlichen verfügt am Ende der Sekundarstufe I nicht über die für ein erfolgreiches berufliches Leben notwendigen Kompetenzen und Wissensstrukturen. Der Hauptschulabschluss so eine Schlussfolgerung aus der Untersuchung - stelle kein hinreichend valides Kriterium zur Bestimmung der Ausbildungsreife dar. Für eine genauere Klärung 
der Bedeutung der in der Sekundarstufe I erworbenen Basiskompetenzen (mathematische Basiskompetenzen, Leseverständnis, Sprachverständnis) für eine erfolgreiche Berufsausbildung fehlt aber derzeit die empirische Basis. Das geplante Berufsbildungs-PISA sollte diesbezüglich Abhilfe schaffen (vgl. Baethge/ Achtenhagen/Arends/Babic/Baethge-Kinsky/Weber 2006).

Die derzeitige Diskussion um „Ausbildungsfähigkeit“ gestaltet sich - auch vor dem Hintergrund einer fehlenden empirischen Untermauerung - äußerst vielfältig, teilweise auch diffus (zu einer Klärung tragen die Beiträge von Eckert, Hilke und Jung in diesem Band bei). Die empirische Bildungsforschung spricht diesbezüglich von einem Set von „Basiskompetenzen“ und „Mindestbildungsstandards". Diese korrelieren mit einer erfolgreichen Einmündung in eine Ausbildung oder mit einer späteren beruflichen Karriere (vgl. Watermann 2003). Noch weitgehend ungeklärt ist zudem die Frage, wie man in der beruflichen Bildung mit so genannten „Risikoschülern“ umgehen soll. Angesichts der zunehmenden Zahl von Lernschwächeren steigt der Druck, ein methodisches Repertoire für diese Zielgruppe zu erarbeiten. Es fehlt an Ansatzpunkten und Beiträgen der beruflich akzentuierten Lehr-Lern-Forschung zu Benachteiligten. Diesbezüglich ist generell ein Desiderat berufsbildender Forschung auszumachen (vgl. Nickolaus/Riedl/Schelten 2005). Ganz ähnlich sieht die Situation übrigens auch für das Phänomen der hochbegabten Auszubildenden aus: Auch diesbezüglich fehlt es an empirischen Untersuchungen und an Konzepten zum Ungang mit dieser Klientel (vgl. Stamm 2006). Dies gilt nicht zuletzt auch für die Frage, wie Lehrkräfte mit der teilweise enormen Heterogenität von Berufsschulklassen umgehen sollen.

Im Folgenden soll die Problematik der mangelnden Ausbildungsfähigkeit unter zwei Aspekten diskutiert werden: Erstens ist zu fragen, welche Folge die Nicht-Teilhabe an beruflichen Sozialisations- und Qualifizierungsprozessen hat. Diesbezüglich wird in Kapitel 2 die These vertreten, dass sich Ungleichheit über die Lebenszeit hinweg verstärkt und dass sich eine ungünstige Startposition nur schwerlich korrigieren lässt. Für die Lehrkräfte vor Ort schließt sich an dieses grundsätzliche gesellschaftliche Problem eine sehr konkrete Fragestellung an: Wie kann sichergestellt werden, dass Lernschwächere in beruflichen Lehr-LernProzessen die für die Bewältigung zukünftiger beruflicher (und privater) Lebenssituationen notwendigen Kompetenzen und Qualifikationen erwerben? Vor dem Hintergrund methodischer Grundentscheidungen (schüler- vs. lehrerzentrierte Arrangements) wird dann in Kapitel 3 gezeigt, wie Lernende mit unterschiedlichen Bildungsabschlüssen die kaufmännische Erstausbildung erleben und welche Lernerfolge sie erzielen. Die Ausführungen schließen mit einem kurzen Fazit. 


\section{Zur Wirkung von Selektionsmechanismen an der ersten Schwelle}

Formal stellen Abschlüsse allgemeinbildender Schulen keine Voraussetzung für den Eintritt in das duale System dar. Dennoch ist der Zugang zu höher bewerteten Ausbildungsberufen de facto an Schulabschlüsse gekoppelt (vgl. Konietzka/ Seibert 2003: 572). Während in den früheren Jahren das duale System insbesondere ein Ausbildungssystem für Hauptschulabsolventen war, ist mittlerweile ein deutlicher Trend in Richtung Höherqualifizierung auszumachen. Abiturienten steht regelmäßig eine Vielfalt von Alternativen offen, für Jugendliche mit bzw. ohne Hauptschulabschluss reduziert sich die Auswahl beträchtlich. Ein Blick auf die Neuabschlüsse von Ausbildungsverträgen differenziert nach Schulbildung zeigt, dass Studienberechtigte vornehmlich in kaufmännischen Berufen zu finden sind. Für Hauptschulabsolventen dagegen wird der Zugang zu zukunftsträchtigen Ausbildungsberufen immer schwieriger. Insbesondere aus kaufmännischer Sicht haben diese Jugendliche vergleichsweise schlechte Karten, hier ist lediglich eine Ausbildung im Einzelhandel eine realistische Option. Die Ausbildungsplatzvergabe selbst ist dann der Ausgangspunkt einer weitgehend vorgegebenen beruflichen Entwicklungsperspektive. Vornehmlich in den 1970er und 1980er Jahren durchgeführte Sozialisationsstudien verweisen eindrücklich auf die Bedeutung der Berufsausbildung für die Persönlichkeitsentwicklung (vgl. Mayer et al. 1981; Häfeli/Kraft/Schallberger 1988). Über Selektionsmechanismen münden vermeintlich schwächere und potenziell benachteiligte Jugendliche in weniger herausfordernde, weniger prestigeträchtige und weniger lukrative Ausbildungsberufe bzw. Tätigkeiten ein. In intellektuell anforderungsreichen Berufen dagegen sammeln sich Jugendliche mit überdurchschnittlicher Leistungsfähigkeit an. Umgekehrt führt die Variation des Anforderungsniveaus zu einer unterschiedlichen Kompetenzentwicklung. Sozialisationseffekte sind die Fortsetzung von Selektionseffekten und vice versa. Es kommt zu einer Scherenentwicklung. Verschärfend wirkt, dass sich Effekte über die Lebenszeit hinweg verstärken bzw. kumulieren. In der Konsequenz ergibt sich eine „Weiterbildungsspirale“ mit der Folge einer Verfestigung der Segmentation (vgl. Münk/Lipsmeier 1997).

\section{Prozessanalysen Selbstorganisierten Lernens}

\subsection{Lehr-Lern-Konzeption}

Bei der Konstruktion einer Lernumgebung steht die Frage nach der mehrkriterialen Wirksamkeit von Unterricht auf dem Prüfstand. Es ist nach komplexen LehrLern-Arrangements zu suchen, mit denen es gelingen kann, gleichzeitig kognitive 
und emotional-motivationale Zielkriterien zu erreichen (vgl. Sembill 1992). Mit der Konzeption des selbstorganisierten Lernens (SoLe) steht ein entsprechender, in der Unterrichtspraxis erprobter Ansatz zur Verfügung. Aufbauend auf reformpädagogischen Ansätzen weist Selbstorganisiertes Lernen eine hohe Affinität zum Projektunterricht im ursprünglichen Sinne auf und zeichnet sich durch eine umfassende Übertragung von Lernverantwortung auf Lernende aus. Zentrale Leitidee des Selbstorganisierten Lernens stellt das (problemlösende) geplante Handeln dar. Es sind vier Lerndimensionen zu unterscheiden: Neben „Lernen für sich“ umfasst das Lehr-Lern-Arrangement „Lernen mit anderen“ (Lernen in Gruppen) sowie „Lernen für andere“ (arbeitsteiliges, verantwortungsbehaftetes Lernen). Die Option „Lernen mit Risiko“ verweist auf die Möglichkeit, Fehler zu machen und aus diesen zu lernen. Dies erfordert von allen Beteiligten ein konstruktives Fehlerverständnis und -management (vgl. Sembill 1992, 2000).

Konkret gesprochen zeichnet sich Selbstorganisierten Lernen durch eine lernerzentrierte und projektorientierte Unterrichtsgestaltung aus, bei der das gemeinsame Lernen in Kleingruppen im Mittelpunkt steht. Im Vergleich zum traditionellen Unterricht verliert die Darbietung von Lerninhalten an Bedeutung. Vielmehr sind etwa 60 bis $70 \%$ der Unterrichtszeit für Eigenaktivitäten der Lernenden reserviert. Die restliche Zeit dient der Hinführung zum Thema, der Ergebnissicherung, der Vertiefung und Wiederholung sowie der Leistungsbeurteilung. Bei der Umsetzung der SoLe-Konzeption empfiehlt es sich, über mehrere Wochen hinweg drei oder vier Unterrichtsstunden zu einer Unterrichtseinheit zusammenzufassen, um genug Zeiträume für die Eigenaktivität der Lernenden zur Verfügung zu stellen. Zudem hat es sich bewährt, das Ausmaß an Eigenverantwortlichkeit und Selbstlernzeit kontinuierlich zu steigern und nicht von Anfang an komplett auf die Eigenaktivität der Lernenden zu setzen. Struktur gewinnt der SoLe-Unterricht zum einen durch eine gemeinsam mit den Lernenden erarbeitete Terminplanung (Meilensteine), durch kurze inhaltliche Inputs und Zusammenfassung der Lehrperson sowie durch den Einsatz von didaktisch entsprechend aufbereiteter Unterrichtsmaterialien.

Zur Prüfung der „Praxistauglichkeit“ der Konzeption wurden im Rahmen dreier Forschungsprojekte („Prozessanalysen Selbstorganisierten Lernens“) in Kooperation mit Lehrkräften entsprechende Lernumgebungen gestaltet:

- Studie 1 („Materialwirtschaft“): Zwei Klassen ( $\mathrm{n}=35), 40$ Unterrichtsstunden, Industriekaufleute (vgl. u.a. Wuttke 1999; Schumacher 2002).

- Studie 2 („Personalwirtschaft“): Zwei Klassen ( $\mathrm{n}=30$ ), 80 Unterrichtsstunden, Bürokaufleute (vgl. u.a. Sembill 2004; Seifried/Klüber 2006).

- Studie 3 („Buchführung“): Zwei Klassen $(\mathrm{n}=45)$, 76 Unterrichtsstunden, kaufmännische Grundstufe (vgl. u.a. Seifried 2004a; 2004b). 
In quasi-experimentellen Feldstudien wurde den Experimentalgruppen (selbstorganisiertes Lernen $=$ SoLe) je eine Kontrollgruppe gegenübergestellt, die vorwiegend nach dem Muster des fragend-entwickelnden Frontalunterrichts beschult wurde (traditionelles Lernen $=$ TraLe). Bei den Auszubildenden wurde die Leistungsentwicklung längsschnittlich durch lernzielorientierte Tests sowie die Bearbeitung von (domänenspezifischen) Problemfällen dokumentiert. Schülerauskünfte zu Motivation und Emotion ergänzen die Datenbasis. Die vor Beginn der eigentlichen Unterrichtsphasen durchgeführte Eingangserhebung dient der Erfassung lernrelevanter Variablen wie z.B. Vorwissen, Motivation sowie biografischer Daten. Der Unterricht wurde jeweils videografiert und die SchülerSchüler-Interaktion aufgezeichnet (siehe Abbildung 1).

Abbildung 1: Schematische Darstellung des Untersuchungsdesigns

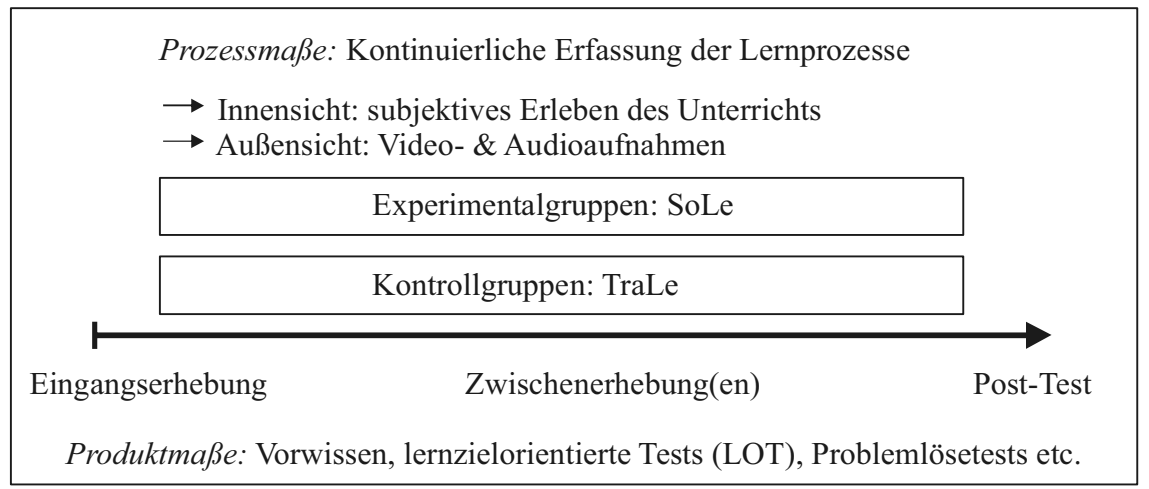

Die empirische Koppelung der Beobachtungs- mit der Erlebensebene ermöglicht die Identifizierung von Zusammenhangsmustern zwischen der sichtbaren Handlungsebene (,Außensicht") und dem nicht unmittelbar beobachtbaren subjektiven Erleben des Unterrichts („Innensicht“). Mit Außensicht sind dabei die beobachtbaren Muster der Unterrichtsorganisation gemeint, wohingegen der Begriff Innensicht die Einschätzungen und Beurteilungen der Schüler bezüglich des Unterrichts beschreibt. Die Berücksichtigung beider Perspektiven eröffnet umfassende Möglichkeiten, Lehr- und Lern-Prozesse im Unterricht näher zu analysieren (vgl. z.B. Seidel 2003: 60ff.). Konkret ergibt sich durch das skizzierte Forschungsdesign die Möglichkeit, Lehr-Lern-Prozesse in Abhängigkeit von situativen Bedingungen zu untersuchen und mehr über die beim Wissenserwerb vermittelnden emotionalen, motivationalen und kognitiven Prozesse zu erfahren. 
Es zeigte sich, dass Schüler in einer selbstorganisationsoffenen Lernumgebung bezüglich Gütekriterien wie Faktenwissen mindestens einen vergleichbaren Lernerfolg erzielen wie Lernende, die eher traditionell unterrichtet werden (vgl. hierzu auch Abschnitt 3.3.1). Darüber hinaus ließ sich feststellen, dass die Schüler bezüglich der von uns als zentral betrachteten Qualifikation „Problemlösekompetenz" sowie, hinsichtlich der Emotionalen Befindlichkeit, Vorteile aufweisen (Produktmaße). Mittels detaillierter Analysen von Lehr-Lern-Prozessen ließen sich eine Vielzahl von Hinweisen finden, die Erklärungsansätze für die Gründe der vielfältigen Überlegenheit der SoLe-Klassen bieten. Diesbezüglich ist insbesondere die gewinnbringende Nutzung der eingeräumten Zeit- und Handlungsfreiräume während des Unterrichts zu nennen (Prozessmaße). Die Darstellung der wesentlichen Ergebnisse der verschiedenen Studien in Abbildung 2 folgt der Unterscheidung zwischen Produkt- und Prozessmaßebene.

Abbildung 2: Zentrale Befunde der Prozessanalysen Selbstorganisierten Lernens (vgl. Sembill/Seifried 2006: 103)

Produktmaßebene:

- SoLe-Schüler/innen konnten signifikant besser als TraLe-Schüler/innen komplexe Probleme in formaler wie qualitativer Weise bearbeiten, obwohl sie teilweise signifikant schlechtere intellektuelle und psychosoziale Voraussetzungen hatten.

- Mit dieser gesteigerten Problemlösefähigkeit ging gleichzeitig bei SoLe-Schüler/ innen eine positive Entwicklung von Interessensbildung und Emotionen einher.

Prozessmaßebene:

- In beiden Lehr-Lern-Arrangements konnte ein enger Zusammenhang von kognitiven, emotionalen und motivationalen Prozessvariablen nachgewiesen werden. Dieser Zusammenhang ist in den SoLe-Klassen allerdings intensiver vernetzt und rückgekoppelt und ist über längere Zeit stabil (Zeitreihenanalysedaten).

- Im vergleichsweise (mit TraLe) gleichen Zeitrahmen werden diese aktiven Lerntätigkeiten signifikant größer und das Lernpotenzial höher.

- SoLe-Schüler/innen erarbeiten sich dabei die Faktengrundlage der Problemlösefähigkeit selbstständig und auf einem höheren Vernetzungsgrad.

- Motor dieses Erarbeitungsprozesses sind Schülerfragen: Lernende im SoLeUnterricht stellen etwa $35 \mathrm{mal}$ so viele Fragen pro Stunde wie Lernende im TraLe-Unterricht. Beachtlich ist auch, dass sie etwa $18 \mathrm{mal}$ so viele Fragen mit deep-reasoning Charakter stellen.

- In diesem Kontext ist $\mathrm{zu}$ beachten, dass SoLe-Schüler/innen einen besseren, nicht extra trainierten Lernstrategieeinsatz mit äquivalenten Auswirkungen auf 


\subsection{Fragestellung und Untersuchungsmethode}

Wie oben bereits ausgeführt, lag der Schwerpunkt der durchgeführten Prozessanalysen Selbstorganisierten Lernens auf dem Vergleich von Experimental- und Kontrollgruppen - auch unter dem Gesichtspunkt fachdidaktischer Besonderheiten. Die hier berichteten Befunde richten den Blick auf Lernschwächere i.w.S. und stellen quasi ein Nebenprodukt unserer Forschungsaktivitäten dar. Zum Problem der an für sich schon kleinen Stichprobe kommt hinzu, dass sich in den von uns untersuchten Klassen kaum Lernschwächere i.e.S. befinden. Alle Schülerinnen und Schüler absolvieren eine kaufmännische Erstausbildung, d.h. alle Probanden waren bei der Ausbildungsstellensuche erfolgreich und haben einen Ausbildungsbetrieb von ihren Qualitäten überzeugt. Sie verfügen mehrheitlich über einen mittleren Bildungsabschluss. Im Folgenden werden zwei Fragestellungen bearbeitet:

1. Welchen Lernerfolg (Faktenwissen und Problemlösekompetenz) erzielen Auszubildende (a) in Abhängigkeit von didaktischen Schwerpunktsetzungen und (b) in Abhängigkeit von der Vorbildung?

2. Differiert das Unterrichtserleben (a) in Abhängigkeit von didaktischen Schwerpunktsetzungen und (b) in Abhängigkeit von der Vorbildung?

Zur Beantwortung der hier aufgeworfenen Fragen erfolgt zunächst ein Rückgriff auf die verschiedenen Leistungsmaße. Zur Feststellung des Vorwissens sollten die Schüler Fragen zum Thema Wirtschaft im Allgemeinen (Items aus dem Wirtschaftskundlichen Bildungstest von Beck und Krumm 1998) und zum jeweiligen Lerninhaltsgebiet im Besonderen (LOT $=$ selbst entwickelte lernzielorientierte Tests auf dem Niveau von Prüfungen der Industrie- und Handelskammern) beantworten. Maximal waren zwölf Punkte für den Themenkomplex „Allgemeine Wirtschaft" und 12 Punkte im lerninhaltsspezifischen Abschnitt zu erzielen. Zur Erfassung des Faktenwissens kamen erneut selbst entwickelte lernzielorientierte Tests zur Anwendung. Hier sollten die Schüler Berechungen durchführen, Buchungssätze bilden, Multiple-Choice-Aufgaben bearbeiten und offene Fragen beantworten. Insgesamt konnten jeweils maximal zwölf Punkte (Studie 1) bzw. sechzehn Punkte (Studie 2 und 3) erreicht werden.

Zur Ermittlung der Problemlösekompetenz wurden den Untersuchungsteilnehmern lerninhaltsspezifische Probleme vorgelegt. Die Auswertung orientiert sich dann an den Schritten einer ,idealen“ Problemlösung (vgl. Dörner 1976; Sembill 1992). Die Schülerlösungen werden hinsichtlich quantitativer Gesichtspunkte ausgewertet. Hierzu zählen neben der Analyse des Ist-Zustands und Zielbzw. Soll-Zustands die Beschreibung möglicher Maßnahmen sowie die Hand- 
lungskontrolle. Diese Aspekte werden anschließend zusammengefasst, gewichtet und zu einem Gesamtkriterium (Analytischer Idealtypus gewichtet) verdichtet. ${ }^{1}$

Das subjektive Erleben des Unterrichts wurde mit Hilfe von mobilen Datenerfassungsgeräten in kurzer zeitlicher Taktung (fünf bzw. zehn Minuten) erhoben. Jeder Schüler gab seine Selbsteinschätzung auf einer stufenlosen Skala von 0 bis 100 bezüglich sechs unterschiedlicher Statevariablen ab, die Aspekte des subjektiven emotionalen, motivationalen und kognitiven Erlebens erfassten. Die drei psychologischen Grunddimensionen wurden wie folgt operationalisiert: Emotion: „Fühle mich ernst genommen“ und „Fühle mich wohl“; Kognition: „Finde Anforderungen hoch“ und „Verstehe, worum es geht"; Motivation: „Ich kann mitgestalten“ und „Bin interessiert“. Bei der Auswahl und Formulierung der Items haben wir uns von der Überlegung leiten lassen, dass eine ausschließlich kognitive Kompetenzbetrachtung des individuellen Prozesses der Leistungserbringung zwangsläufig zu kurz greift. Das Aktivieren eines Handlungsschemas ist vielmehr immer auch das Ergebnis von Bewertungsprozessen und damit von Emotionen. Emotional-motivationale Prozesse sind also immer in zentraler Weise an der Steuerung kognitiver Handlungsregulations-Prozesse beteiligt (vgl. Seifried/Sembill 2005).

Zur Erfassung der didaktischen Schwerpunktsetzungen wurde der Unterricht auf Video aufgezeichnet und anschließend entsprechend kodiert. Hinsichtlich der Kategorie „unterrichtliche Arbeitsform“ beispielsweise wurde zwischen Frontalunterricht und schülerzentrierten Arbeitsphasen differenziert. Letztere sind dadurch gekennzeichnet, dass Lehrpersonen nach dem Bereitstellen der Unterrichtsmaterialien nur noch einen vergleichsweise geringen Einfluss auf die Lerntätigkeit nehmen, wohingegen im Frontalunterricht eine lehrende Person (dies kann z.B. im Rahmen von Ergebnispräsentationen auch ein Schüler sein) den Unterrichtsablauf steuert und kontrolliert.

1 Um zu verhindern, dass eine ausführliche Aufzählung von Ist-Zuständen fehlende Aussagen zu Maßnahmen oder zur Handlungskontrolle kompensiert, erfolgt eine Gewichtung der einzelnen Kategorien. Hierzu wird über alle Untersuchungsteilnehmer hinweg das Komplement zu dem beobachtbaren Anteil einer Kategorie an der Summe aller kodierten Aussagen gebildet. Oft gebrauchte Kategorien erhalten dadurch ein geringes Gewicht, weniger frequentierte Kategorien sind dementsprechend mit einem hohen Gewichtungsfaktor zu versehen. 


\subsection{Empirische Befunde}

\subsubsection{Lernerfolg}

In einem ersten Analyseschritt wird getrennt für die drei Studien dargestellt, wie die Schülerinnen und Schüler mit unterschiedlichen Bildungsniveaus bei den Leistungstests abschneiden (vgl. Tabelle 1).

Tabelle 1: $\quad$ Treatment, Schulabschlüsse und Lernleistung

\begin{tabular}{|c|c|c|c|c|c|c|c|c|c|c|}
\hline \multirow{2}{*}{$\begin{array}{l}\text { Treatment } \\
\text { Vorbildung }\end{array}$} & \multicolumn{5}{|c|}{ SoLe } & \multicolumn{5}{|c|}{ TraLe } \\
\hline & GSP & HS & RS & WS & GYM & GSP & HS & RS & WS & GYM \\
\hline \multicolumn{11}{|c|}{ Studie 1 (Materialwirtschaft) } \\
\hline $\mathrm{N}$ & 21 & --- & 12 & --- & 9 & 14 & --- & 1 & --- & 13 \\
\hline Vorwissen (WBT, LOT) & 6,9 & -- & 7,3 & -- & 6,2 & 6,6 & -- & 7,0 & --- & 6,2 \\
\hline Faktenwissen (LOT) & 4,5 & --- & 4,4 & --- & 4,7 & 4,4 & --- & 5,0 & --- & 4,4 \\
\hline Problemlösung (AITG) & 6,9 & --- & 5,5 & --- & 8,7 & 4,7 & --- & 2,1 & --- & 4,9 \\
\hline \multicolumn{11}{|c|}{ Studie 2 (Personalwirtschaft) } \\
\hline $\mathrm{N}$ & 15 & 1 & 12 & --- & 2 & 15 & 1 & 12 & --- & 2 \\
\hline Vorwissen (WBT, LOT) & 6,1 & 3,5 & 6,0 & -- & 7,5 & 5,0 & 2,0 & 5,5 & --- & 4,3 \\
\hline Faktenwissen (LOT) & 6,2 & 4,3 & 6,1 & --- & 7,7 & 5,9 & 3,0 & 6,0 & --- & 6,5 \\
\hline Problemlösung (AITG) & 6,8 & 1,0 & 7,5 & --- & 5,8 & 5,3 & 2,1 & 5,7 & --- & 4,3 \\
\hline \multicolumn{11}{|l|}{ Studie 3 (Buchführung) } \\
\hline $\mathrm{N}$ & 22 & 6 & 14 & 2 & --- & 23 & 7 & 10 & 4 & 2 \\
\hline Vorwissen (WBT, LOT) & 6,4 & 4,1 & 7,1 & 8,8 & $\begin{array}{ll}-- \\
---\end{array}$ & 8,6 & 7,4 & 8,6 & 9,1 & 10,8 \\
\hline Faktenwissen (LOT) & 9,5 & 7,7 & 10,0 & 11,6 & --- & 9,1 & 6,6 & 9,9 & 10,1 & 12,3 \\
\hline Problemlösung (AITG) & 8,0 & 6,1 & 9,0 & 7,4 & --- & 6,6 & 5,0 & 6,9 & 8,0 & 8,9 \\
\hline
\end{tabular}

Anmerkung: Die Werte in der Tabelle sind Mittelwerte. Beim Vorwissenstest können maximal 26 Punkte erreicht werden, beim Test auf Faktenwissen 12 (Studie 1) bzw. 16 Punkte (Studie 2 und 3). Beim Problemlösetest existiert kein festgelegter Maximalwert, die Erfahrung zeigt aber, dass i. d. R. bestenfalls 20 Punkte möglich sind.

GSP: Gesamtstichprobe, HS: Hauptschule, RS: Realschule, WS: Wirtschaftsschule, GYM: Gymnasium, WBT $=$ Wirtschaftskundlicher Bildungstest, LOT: lernzielorientierter Test, AITG: Analytischer Idealtypus (gewichtet).

Für Studie 1 zeigt sich, dass SoLe-Schüler beim Faktenwissen einen vergleichbaren Leistungsstand wie TraLe-Schüler erreichen. Zudem verfügen die SoLeSchüler über signifikante Vorteile hinsichtlich der Problemlösekompetenz. Be- 
merkenswert ist u.a., dass die SoLe-Realschüler das Niveau der TraLe-Gymnasiasten erreichen. Darüber hinaus besteht ein tendenzieller Zusammenhang zwischen Vorwissen und Lernerfolg (Faktenwissen: $r=.33$ ). Ähnlich stellt sich die Situation für Studie 2 dar: Erneut erreichen SoLe-Schüler beim Faktenwissen einen vergleichbaren Leistungsstand wie TraLe-Schüler. Und erneut lassen sich für die SoLe-Schüler signifikante Vorteile hinsichtlich der Problemlösekompetenz feststellen. Schließlich besteht ein überzufälliger Zusammenhang zwischen Vorwissen und Lernerfolg (Faktenwissen: $r=.41^{*}$; Problemlösefähigkeit: $r=.48^{* *}$ ). Vor diesem Hintergrund vermögen die Ergebnisse der dritten Studien nunmehr kaum zu überraschen: Wiederum erreichen SoLe-Schüler beim Faktenwissen einen vergleichbaren Leistungsstand wie TraLe-Schüler, und wiederum verzeichnen SoLe-Schüler signifikante Vorteile hinsichtlich der Problemlösekompetenz. Auch hier besteht ein signifikanter Zusammenhang zwischen Vorwissen und Lernerfolg (Faktenwissen: $\mathrm{r}=.62 * *$; Problemlösefähigkeit: $\mathrm{r}=.44 * *$ ).

\subsubsection{Subjektives Erleben des Unterrichts}

Im Rahmen weiterführender Analysen stellt sich nun die Frage, inwieweit sich für verschiedene „activity structures“ (Berliner 1983) innerhalb des jeweiligen Lehr-Lern-Arrangements Unterschiede ergeben. Zu vermuten ist, dass Schüler in Einzel- oder Gruppenarbeitsphasen eine höhere Aktivierung aufweisen als im Frontalunterricht. Dies wird in einschlägigen didaktischen Kompendien (z.B. Meyer 1994) regelmäßig postuliert; nach wie vor mangelt es jedoch an empirischen Untersuchungen, die sich konkret mit den motivationalen und kognitiven Auswirkungen verschiedener Arbeitsformen auseinander setzen (zu Ausnahmen siehe bspw. Wild 2000; Seidel 2003). Weiterhin ist von Interesse, ob das Unterrichtserleben in den verschiedenen Unterrichtsphasen von der individuellen Konstitution der Lernenden beeinflusst wird. Unter Rückgriff auf Ergebnisse aus der Aptitude-Treatment-Interaction-Forschung ${ }^{2}$ ist $\mathrm{zu}$ vermuten, dass Schüler mit niedrigerem Vorwissen und hohem Angstniveau eher von den klaren Strukturen des traditionellen Unterrichts und leistungsstärkere und weniger ängstliche Schüler von offenem Unterricht profitieren. In der Lehr-Lern-Forschung ergeben sich diesbezüglich jedoch widersprüchliche Befunde. Während beispielsweise Nickolaus \& Bickmann (2002) für handlungsorientiert unterrichtete schwache Klassen

2 Die ATI-Forschung beschäftigt sich mit der Wechselwirkung von individuellen Lernervoraussetzungen und Lehrmethoden. Ein Forschungsergebnis ist beispielsweise, dass schwächere Lerner von einer klaren Strukturierung des Unterrichts profitieren (Lernen mit Anleitung), wohingegen stärkeren Schülerinnen und Schüler eine Öffnung des Unterrichts entgegenkommt (Lernen ohne Anleitung). 
Nachteile konstatieren, ergeben sich im Rahmen der Münchner Hauptschulstudie und Grundschulstudie keine derartigen Effekte (Helmke/Weinert 1997: 142).

Wie oben bereits ausgeführt, wurde das Unterrichtserleben in kurzer zeitlicher Taktung erfasst (Skala von 0 bis 100). Im Vorfeld wird zunächst pro Untersuchungsteilnehmer für jedes Erlebensitem ein Kennwert in Abhängigkeit von der jeweiligen Arbeitsform (Frontalunterricht vs. schülerzentrierte Arbeitsphase) ermittelt. Zur Ermittlung möglicher Unterschiede dient dann für jede Kategorie eine zweifaktorielle Varianzanalyse mit einem Messwiederholungsfaktor (unterrichtliche Arbeitsform: Frontalunterricht vs. schülerzentrierte Arbeitsphasen) und einem unabhängigen Faktor (Treatment: SoLe vs. TraLe). Im Folgenden werden die Befunde aus der Studie 2 (Personalwirtschaft) für das motivationale Erleben dargestellt (Items: „Bin interessiert“ und „Ich kann mitgestalten“). ${ }^{3}$

Abbildung 3: Prozesserleben in Abhängigkeit von didaktischen Schwerpunktsetzungen und der unterrichtlichen Arbeitsform (vgl. Seifried/ Klüber 2006, 13)

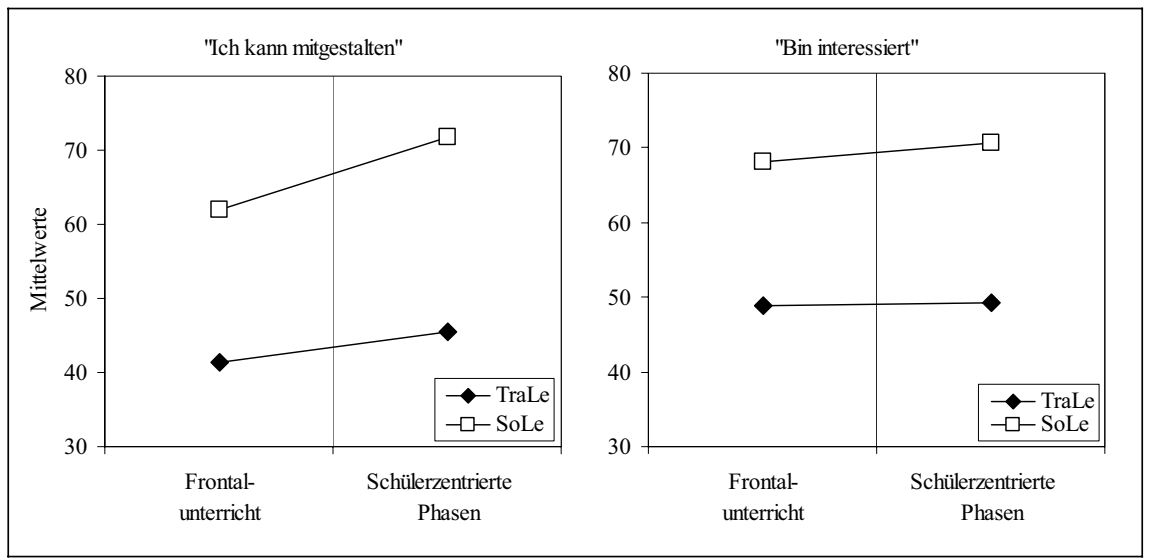

Wie man Abbildung 3 entnehmen kann, liegt sowohl in der SoLe- als auch in der TraLe-Klasse das wahrgenommene Ausmaß an Mitgestaltungsmöglichkeiten während der schülerzentrierten Arbeitsphasen über den Werten für Frontalunterricht. Der Haupteffekt für den Faktor „unterrichtliche Arbeitsform“ ist hoch signifikant und substanziell $\left(\mathrm{F}=13.858, \mathrm{p}=.001, \eta^{2}=.409\right)$. Im Ausmaß etwas geringer, aber immer noch statistisch bedeutsam ist der Effekt für das berichtete

3 Weiterführende Hinweise finden sich in Seifried \& Klüber 2006, Informationen zu vergleichbaren Befunde aus Studie 3 (Buchführung) kann man Seifried 2005 entnehmen. 
Interesse $\left(F=3.224, p=.088, \eta^{2}=.139\right)$. Erneut bewegen sich die Mittelwerte in den schülerzentrierten Unterrichtssegmenten über den Vergleichsgrößen für Frontalunterricht, und erneut stellt sich die Situation für die SoLe-Klasse deutlich positiver dar als für die TraLe-Klasse.

Anschließend wird überprüft, wie sich das Vorwissen der Schüler auf das subjektive Erleben der beiden Lehr-Lern-Situationen auswirkt. Mittels Mittelwertsplit wurden die Untersuchungsteilnehmer in zwei Gruppen mit niedrigem vs. hohem Vorwissen eingeteilt. Zur Ermittlung möglicher Effekte dient erneut eine zweifaktorielle Varianzanalyse mit einem Messwiederholungsfaktor (unterrichtliche Arbeitsform: Frontalunterricht vs. schülerzentrierte Arbeitsphasen) und einem unabhängigen Faktor (Vorwissen: hoch vs. gering) (vgl. Abbildung 4). Bei dieser Teilanalyse ergibt sich für „Ich kann mitgestalten“ ein Haupteffekt in Abhängigkeit von der unterrichtlichen Arbeitsform $\left(F=11.855, p=.003, \eta^{2}=\right.$ 397) sowie ein Wechselwirkungseffekt zwischen Vorwissensniveau und unterrichtlicher Arbeitsform $\left(\mathrm{F}=8.237, \mathrm{p}=.010, \eta^{2}=.314\right)$. Insbesondere Lernende mit Nachteilen hinsichtlich des Vorwissens berichten über ein höheres Ausmaß des Mitgestaltens in schülerzentrierte Arbeitsphasen. Offensichtlich führt im vorliegenden Fall eine lehrerzentrierte Vorgehensweise vornehmlich bei Lernern ohne (umfangreiche) Vorkenntnisse zum Gefühl der Passivität, wohingegen - in der Wahrnehmung der Befragten - das Autonomieniveau in Phasen des Gruppen- bzw. Projektunterrichts sehr viel stärker ausgeprägt ist.

Abbildung 4: Prozesserleben in Abhängigkeit vom Vorwissen und der unterrichtlichen Arbeitsform (vgl. Seifried/Klüber 2006: 16)

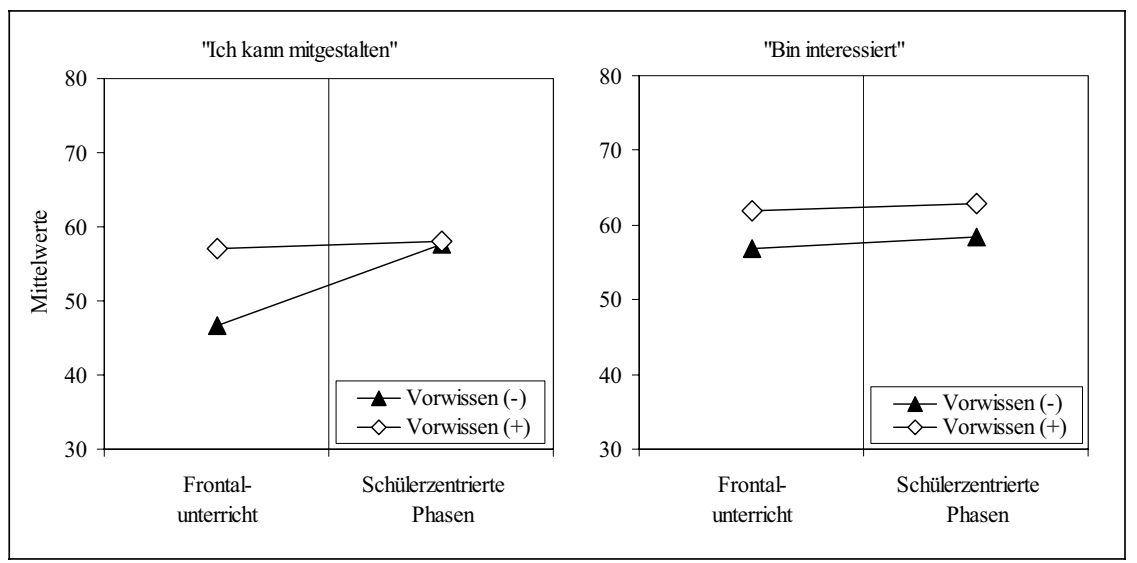




\section{$4 \quad$ Fazit}

Im empirischen Teil des Beitrags wurde überprüft, inwiefern Lernende mit unterschiedlicher Vorbildung mit verschiedenen Lehr-Lern-Arrangements zurechtkommen. Angesichts des geringen Stichprobenumfangs sind die Befunde mit der gebotenen Zurückhaltung zu interpretieren. Es hat sich jedoch in jeder der Studien gezeigt, dass Lernschwächere offenbar keine Nachteile erleiden, wenn sie in Form des selbstorganisierten Lernens beschult werden. Die Befürchtung, lernschwächere Schüler durch offene Unterrichtsarrangements systematisch zu überfordern, hat sich im vorliegenden Fall nicht bewahrheitet. Im Gegenteil: Schwächere und stärkere Schüler profitieren jeweils individuell von der Öffnung des Unterrichts und erreichen jeweils bessere Ergebnisse als die Lernenden der entsprechenden Leistungsklassen der Kontrollgruppen. Es wird zudem herausgestellt, dass das Vorwissen eine zentrale Größe für die Vorhersage der Leistungsentwicklung darstellt.

Bei der Analyse des subjektiven Erlebens des Unterrichts ergeben sich eine Reihe theoriekonformer Resultate: Es bestehen erwartungsgemäß Wahrnehmungsunterschiede in Abhängigkeit vom Treatment und in Abhängigkeit von der unterrichtlichen Arbeitsform. Die Lernenden berichten treatmentübergreifend in Phasen selbstständiger Schülerarbeit über ein höheres Ausmaß an Mitgestaltungsmöglichkeiten als in lehrerzentrierten Unterrichtsphasen. Dieses Resultat erscheint auf den ersten Blick trivial. Die im Vergleich zum Frontalunterricht höheren Werte bei „Ich kann mitgestalten“ im Rahmen schüleraktiver Unterrichtsphasen weisen jedoch darauf hin, dass die Lernenden bestehende Freiheitsgrade auch als solche wahrnehmen, und dies im doppelten Wortsinne. Das höhere Ausmaß an Mitgestaltungsmöglichkeiten spiegelt sich theoriekonform auch in den Selbstberichten bezüglich des aktuellen Interesses wider: Die Schülervoten während der schülerzentrierten Arbeitsphasen liegen über den Vergleichswerten für den Frontalunterricht. Die berichteten Zusammenhänge sind daher als weiteres Indiz für die motivationsfördernde Wirkung einer aktiven und selbstbestimmten Auseinandersetzung mit Lerninhalten zu werten. Die theoretisch begründeten Annahmen über systematische Zusammenhänge zwischen motivationsrelevanten Bedingungen und dem motivationalen Erleben des Unterrichts werden durch die vorliegenden Befunde somit unterstützt.

Bezüglich der Analyse des subjektiven Erlebens von Lehr-Lern-Situationen in Abhängigkeit von Vorwissen lässt sich keine Bestätigung der Ergebnisse der Aptitude-Treatment-Interaction-Forschung finden. Für Lernende mit Nachteilen hinsichtlich des lerninhaltsspezifischen Vorwissens ergeben sich überzufällige Vorteile zugunsten des schülerzentrierten Unterrichts. So berichten diese Schüler in Phasen selbstständiger Bearbeitung von Lerninhalten über ein höheres Ausmaß 
an Mitgestaltungsmöglichkeiten als in lehrerzentrierten Unterrichtsphasen. Die Interpretation weiterer Erlebensdaten (vgl. hierzu Seifried/Klüber 2006) unterstützen die Vermutung, dass Schüler mit geringerem Vorwissen im Frontalunterricht nicht ausreichend partizipieren können. Umgekehrt scheinen Lernen mit einem profunden Vorwissen auch im TraLe-Unterricht ausreichende Möglichkeiten der Partizipation zu finden. ${ }^{4}$ Die Befunde der Analyse der Lernergebnisse weisen in eine ähnliche Richtung wie die eben skizzierte Auswertung der Erlebensdaten: Während TraLe-Schüler mit niedrigem Vorwissen bezüglich des Faktenwissens im Zeitverlauf nicht das Niveau der Schüler mit hohem Vorwissen erreichen, können SoLe-Schüler mit niedrigem Vorwissen die zu Untersuchungsbeginn bestehenden Nachteile deutlich besser ausgleichen (vgl. Sembill 2004).

In der Förderung der Eigenaktivität von Lernenden scheint also ein Schlüssel zum Umgang mit heterogenen Lerngruppen zu liegen. Vor dem Hintergrund der vorliegenden Befunde empfehlen wir daher eine konsequente Ausweitung der Handlungs- und Schülerorientierung. Eine entsprechend angelegte Qualifizierung sollte auch für lernschwächere Schülerinnen und Schüler eine erfolgversprechende Option darstellen. Lehrpersonen sind also aufgefordert, Lehr-LernProzesse ganzheitlich zu betrachten und den Lerner mit seinen individuellen Stärken und Schwächen konsequent in das Zentrum der pädagogischen Bemühungen zu rücken. Hierzu bedarf es einer umfassenden Neukonzeption des gesamten Unterrichts: Schüler und Lehrer gestalten gemeinsam die Lehr-LernProzesse so, dass im Vergleich zur herkömmlichen Qualifizierung ein größeres Ausmaß an Selbst- und Mitbestimmung, die zwingend auch mit der Selbstverantwortung und entsprechenden Beurteilungsprozessen zu koppeln sind, erreicht wird.

\section{Literatur}

Badel, S. (2003): Basiskompetenzen von Schülerinnen und Schülern in berufsvorbereitenden Maßnahmen des Berufsfeldes Ernährung und Hauswirtschaft in Berlin. In Buer, J. van; Zlatkin-Troitschanskaia, O. (Hrsg.): Berufliche Bildung auf dem Prüfstand. Entwicklung zwischen systemischer Steuerung, Transformation durch Modellversuche und unterrichtlicher Innovation. Frankfurt/Main et al.: Lang: 119-156.

Baethge, M.; Achtenhagen, F.; Arends, L.; Babic, E.; Baethge-Kinsky, V.; Weber, S. (2006): Berufsbildungs-PISA. Machbarkeitsstudie. Stuttgart: Franz Steiner.

Beck, K.; Krumm, V. (1998): Wirtschaftskundlicher Bildungs-Test. Handanweisung. Göttingen: Hogrefe.

4 Um diesbezüglich weitere Aussagen treffen zu können, müsste man anhand der Videoaufzeichnungen die vorherrschenden Lehrer-Schüler-Interaktionsmuster unter dem Aspekt „Beteiligungsgerechtigkeit und Lernchancenzuweisung im Frontalunterricht“ näher analysieren. 
Berliner, D. C. (1983): Developing conceptions of classroom environments: Some light on the $\mathrm{t}$ in classroom studies of ATI. Educational Psychologist, 18(1), 1-13.

Dörner, D. (1976): Problemlösen als Informationsverarbeitung, Stuttgart: Kohlhammer.

Fend, H. (1981): Theorie der Schule. 2., durchges. Aufl. München, Wien, Baltimore: Urban \& Schwarzenberg.

Häfeli, K.; Kraft, U.; Schallberger, U. (1988): Berufsausbildung und Persönlichkeitsentwicklung. Eine Längsschnittstudie. Bern, Stuttgart; Toronto: Hans Huber.

Helmke, A.; Weinert, F. E. (1997): Bedingungsfaktoren schulischer Leistungen. In: Weinert, F. E. (Hrsg.): Enzyklopädie der Psychologie, Band 3 (Psychologie der Schule und des Unterrichts). Göttingen: Hogrefe, 71-176.

Konietzka, D.; Seibert, H. (2003): Deutsche und Ausländer an der „zweiten Schwelle“. Eine vergleichende Analyse der Berufseinstiegskohorten 1976-1995 in Westdeutschland. Zeitschrift für Pädagogik, 49(4), 567-590.

Mayer, E.; Schumm, W.; Flaake, K.; Gerberding, H.; Reuling, J. (1981): Betriebliche Ausbildung und gesellschaftliches Bewusstsein. Die berufliche Sozialisation Jugendlicher. Frankfurt a. M.; New York: Campus.

Meyer, H. (1994): Unterrichtsmethoden. 1. Theorieband. Frankfurt/Main: Cornelsen Scriptor.

Münk, D.; Lipsmeier, A. (1997): Berufliche Weiterbildung. Grundlagen und Perspektiven im nationalen und internationalen Kontext. Baltmannsweiler: Schneider-Verlag Hohengehren.

Nickolaus, R.; Bickmann, J. (2002): Kompetenz- und Motivationsentwicklung durch Unterrichtskonzeptionsformen. Erste Ergebnisse einer empirischen Untersuchung bei Elektroinstallateuren. Die Berufsbildende Schule, 54 (7-8), 236-243.

Nickolaus, R.; Riedl, A.; Schelten, A. (2005): Ergebnisse und Desiderate zur LehrLernforschung in der gewerblich-technischen Berufsausbildung. Zeitschrift für Berufs- und Wirtschaftspädagogik, 101(4), 507-532.

Schumacher, L. (2002): Emotionale Befindlichkeit und Motive in Lerngruppen. Hamburg: Dr. Kovač.

Seidel, T. (2003): Lehr-Lernskripts im Unterricht: Freiräume und Einschränkungen für kognitive und motivationale Lernprozesse - eine Videostudie im Physikunterricht. Münster, New York, München, Berlin: Waxmann.

Seifried, J. (2004a): Fachdidaktische Variationen in einer selbstorganisationsoffenen Lernumgebung - Eine empirische Untersuchung im Rechnungswesenunterricht. Wiesbaden: Deutscher Universitätsverlag.

Seifried, J. (2004b): Der Einfluss didaktischer Schwerpunktsetzungen auf das motivationale Erleben des Rechnungswesenunterrichts in einer selbstorganisationsoffenen Lernumgebung. Zeitschrift für Berufs- und Wirtschaftspädagogik, 100(2), 242-255.

Seifried, J. (2005): Lernmotivation in lehrer- und schülerzentrierten Unterrichtssequenzen - Analyse des Unterrichtserlebens mit Hilfe von Selbstberichts- und Videodaten. In: Gonon, P.; Klauser, F.; Nickolaus, R.; Huisinga, R. (Hrsg.): Kompetenz, Kognition und neue Konzepte der beruflichen Bildung. Schriftenreihe der Sektion Berufs- und Wirtschaftspädagogik der DGfE. Wiesbaden: VS Verlag für Sozialwissenschaften, 237-251.

Seifried, J.; Klüber, C. (2006): Unterrichtserleben in schüler- und lehrerzentrierten Unterrichtsphasen. Unterrichtswissenschaft, 34(1), 2-21. 
Seifried, J.; Sembill, D. (2005): Emotionale Befindlichkeit in Lehr-Lern-Prozessen in der beruflichen Bildung. Zeitschrift für Pädagogik, 51(5), 656-672.

Sembill, D. (1992): Problemlösefähigkeit, Handlungskompetenz und Emotionale Befindlichkeit. Zielgrößen Forschenden Lernens. Göttingen, Toronto, Zürich: Hogrefe.

Sembill, D. (2000): Selbstorganisiertes und Lebenslanges Lernen. In: Achtenhagen, F.; Lempert, W. (Hrsg.): Lebenslanges Lernen - seine Grundlegung im Kindes- und Jugendalter, Band 4: Formen und Inhalte von Lernprozessen. Opladen: Leske und $\mathrm{Bu}-$ drich, 60-90.

Sembill, D. (2004): Abschlussbericht zu „Prozessanalysen Selbstorganisierten Lernens“ im Rahmen des DFG-Schwerpunktprogramms „Lehr-Lern-Prozesse in der kaufmännischen Erstausbildung“. Bamberg: Verlag.

Sembill, D.; Seifried, J. (2006): Selbstorganisiertes Lernen als didaktische Lehr-LernKonzeption zur Verknüpfung von selbstgesteuertem und kooperativem Lernen. In: Euler, D.; Pätzold, G.; Lang, M. (Hrsg.): Selbstgesteuertes Lernen in der beruflichen Bildung. Beiheft 20 zur Zeitschrift für Berufs- und Wirtschaftspädagogik. Stuttgart: Franz Steiner, 93-108.

Stamm, M. (2006): Hoch begabte Lehrlingen: eine soziale Tatsache? Zeitschrift für Erziehungswissenschaft, 9(1), 127-139.

Watermann, R. (2003): Ausbildungsreife - Ergebnisse der neuen empirischen Bildungsforschung. Quelle: http://www.bibb.de/dokumente/pdf/a21_ft-zukunf-berufsausbildung watermann.pdf 20.01.2007.

Wild, K.-P. (2000): Der Einfluss von Unterrichtsmethoden und motivationale Orientierungen auf das kognitive Engagement im Berufsschulunterricht. In: Duit, R.; Rhöneck, C. von (Hrsg.): Ergebnisse fachdidaktischer und psychologischer Lehr-LernForschung. Beiträge zu einem Workshop an der Pädagogischen Hochschule Ludwigsburg. Kiel: IPN, 35-54.

Wuttke, E. (1999): Motivation und Lernstrategien in einer selbstorganisationsoffenen Lernumgebung. Eine empirische Untersuchung bei Industriekaufleuten. Frankfurt/ Main et al.: Lang. 